Paper:

\title{
Adaptive Active Vibration Control for Machine Tools with Highly Position-Dependent Dynamics
}

\author{
Robin Kleinwort ${ }^{\dagger}$, Jonathan Platz, and Michael F. Zaeh \\ Technical University of Munich \\ Boltzmannstrasse 15, 85748 Garching, Germany \\ ${ }^{\dagger}$ Corresponding author, E-mail: Robin.Kleinwort@iwb.mw.tum.de \\ [Received November 29, 2017; accepted June 5, 2018]
}

\begin{abstract}
The material removal rates of machine tools are often limited by chatter, which is caused by the machine's most flexible structural modes. Active vibration control systems mitigate chatter vibrations and increase the chatter free axial depth of cut. However, modelbased control strategies reach their limit if the machine tool exhibits highly position-dependent dynamics. In this paper, an adaptive control strategy is presented. This strategy uses online system identification to adapt the controller. The adaption algorithm is mainly automated. However, a few parameters still need to be selected. Therefore, a methodology for the determination of the optimal parameters is proposed. The adaptive controller was implemented on a $B \& R$ PLC and its suitability was verified experimentally by the observation of notable increases in the chatter-free material removal rates.
\end{abstract}

Keywords: chatter, vibrations, active damping, adaptive controller

\section{Introduction}

Chatter vibrations occur under certain machining conditions and lead to poor surface finish and large dynamic forces that can damage the machine and the part [1]. Active vibration control (AVC) systems mitigate chatter vibrations related to the natural modes of the machine tool's structure, and therefore lead to increased precision, increased material removal rate, increased tool life, and decreased cost by avoiding corrective work [2]. Different actuator designs exist, depending on the application [1]: active spindle bearings [3], active tools [4], active workpiece holders [5], and active structural chatter suppression using an inertial actuator [6-8]. In this study, an AVC system with an inertial actuator was used. As reported in [8], model-based control strategies perform better with regard to chatter mitigation, in comparison with the frequently used non-model-based direct velocity feedback controller. However, even robust model-based control strategies reach their limit if the dynamic behavior is highly dependent on position. Adaptive control strategies,

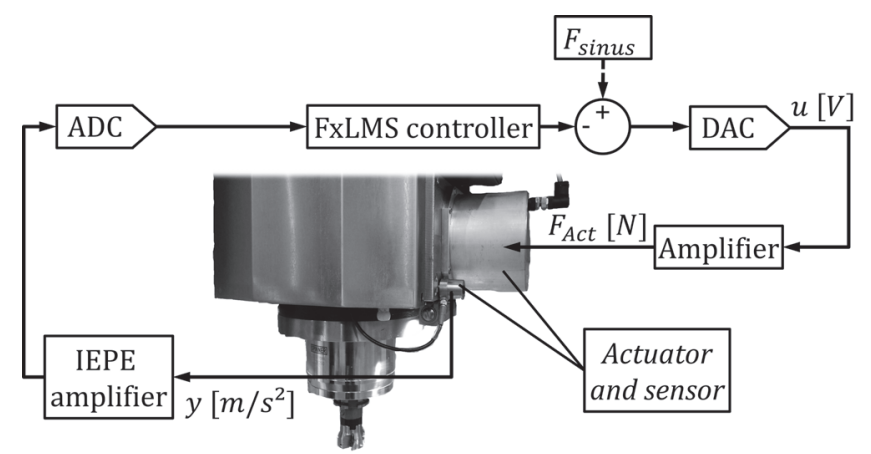

Fig. 1. Experimental setup of the AVC system.

such as the filtered-x least mean square (FxLMS) algorithm used in this study, have been developed for control objects that vary [9], and are therefore able to react to the machine tools' dynamics changes. The FxLMS algorithm has already been tested successfully in the active vibration control of machine tools for composite machining [10] and metal machining [11, 12] by using piezoelectric actuators. In this study, the FxLMS algorithm was used with online system identification, which enables active vibration control for strong position dependent dynamics. Additionally, the algorithm was extended by limiting the controller output, which allowed for the use of the FxLMS algorithm with an easy to attach electromagnetic inertial actuator without risking the occurrence of saturation related damage.

\section{Experimental Setup}

The experimental setup of the AVC system is shown in Fig. 1. An accelerometer (KS813b by MMF) was attached to the $z$-axis of the SPINNER U5-620 5-axis machining center. The measured vibration signal $y(t)$ was acquired through an IEPE amplifier with a built-in 2nd order Butterworth low-pass filter with a cut-off frequency of $1 \mathrm{kHz}$. On a B\&R X20CP1586 PLC the controller was implemented with a sampling frequency of $2.5 \mathrm{kHz}$. The PLC was extended by the X20AI2222 analog input module, and the X20AO4622 analog output module. To 


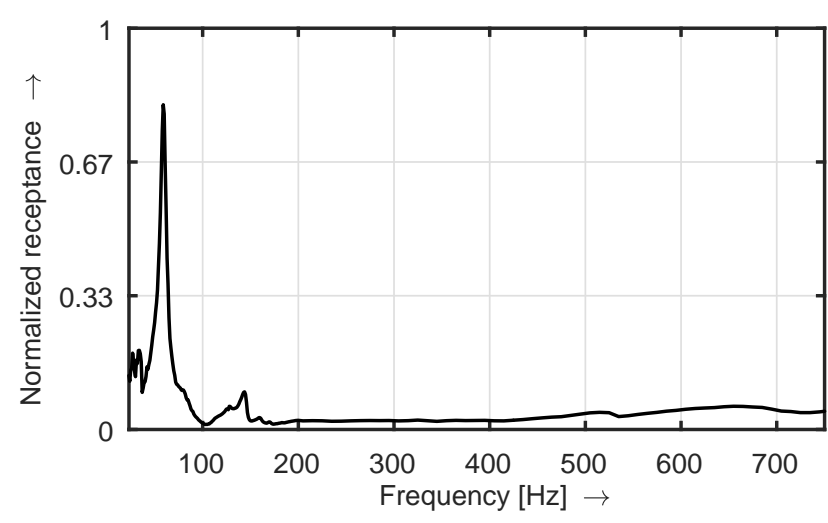

Fig. 2. Measured direct FRF at the TCP in $x$-direction of the SPINNER U5-620.

test the controller, a sinusoidal signal $\left(F_{\text {sinus }}\right)$ was applied within the PLC. The resulting PLC output signal $u(t)$ was amplified (BAA 1000 by BEAK) and sent to the electromagnetic actuator (SA10-V30 by CSA Engineering), which applied a force $\left(F_{A c t}(t)\right)$ to the machine's structure. The nominal force output is $45 \mathrm{~N}$ at a bandwidth of $20-1000 \mathrm{~Hz}$ and the maximum peak force was limited to $90 \mathrm{~N}$. The actuator was placed close to the sensor at an antinode location of the machine's structural mode at $58 \mathrm{~Hz}$, which had to be damped. Fig. 2 shows the receptance frequency response function (FRF) at the tool center point (TCP). For confidentiality reasons, the measured values were normalized to the value of one.

\section{Offline Modeling of Secondary Path}

In this section, a brief summary of the general offline secondary path modeling setup is provided based on [13, 14].

\subsection{General Setup}

To successfully cancel any unwanted vibrations, the FxLMS algorithm must be provided with a model of the secondary path. This model can be determined prior to the control implementation with an offline identification setup using the LMS algorithm. Fig. 3 shows the respective system identification structure. The excitation signal $x(n)$, which is selected by the user, is transmitted to the actuator, finite impulse response (FIR) filter $W(z)$, and LMS algorithm. The filter output $y(n)$ is intended to cancel the signal $d(n)$, which is created by the transmission behavior of the secondary path $S(z)$ and is measured by the acceleration sensor. The modeling error $e(n)$ is fed through to the LMS algorithm, which is designed to minimize $e(n)$ by adapting the FIR filter weights. Thus, $W(z)$ is shaped after the secondary path $S(z)$ and can be employed as a system model as soon as the identification process has been completed. In this study, the normalized LMS algorithm was used. This algorithm adapts the filter weights

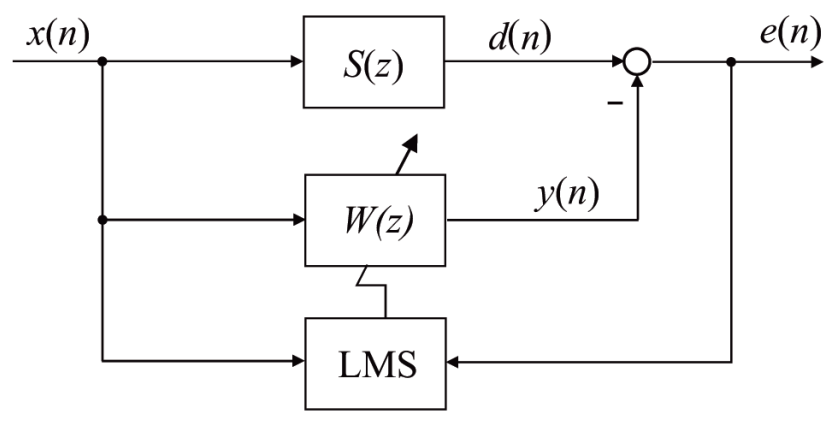

Fig. 3. Offline identification setup using LMS algorithm.

according to the following equation:

$$
\boldsymbol{w}(n+1)=\boldsymbol{w}(n)+\frac{\alpha}{L P_{x}(n)} \boldsymbol{x}(n) e(n), \quad . \quad . \quad .
$$

where $\boldsymbol{w}(n)$ is the filter weight vector, $\alpha$ is the adaptation step size, and $L$ is the number of filter weights, respectively. Vector $\boldsymbol{x}(n)$ contains the latest $L$ samples of the reference signal $x(n)$. Its signal power is expressed as follows:

$$
P_{x}(n)=\frac{1}{L} \boldsymbol{x}^{T}(n) \boldsymbol{x}(n) .
$$

To prevent the fractional expression in Eq. (1) from becoming extremely large in the case of a very low signal power $\left(P_{x}(n)\right)$, it is sensible to employ a lower threshold $\left(P_{x, \text { min }}\right)$.

Regarding the choice of excitation signal, the signal spectrum must contain all of the relevant frequencies. Hence, the most sensible choice is generally band-limited white noise, owing to its flat spectrum across all frequencies.

\subsection{Determination of Optimal LMS Parameters}

To determine the optimal LMS parameters for offline identification, various simulations were conducted. During the simulations, the most influential LMS parameters were varied systematically and the respective parameter set was reviewed based on the accordance between the model identified by the LMS algorithm and a previously measured frequency response function (FRF). The degree of accordance was quantified by using the frequency amplitude assurance criterion (FAAC [15]), whose value ranged from zero (no accordance) to one (perfect accordance), and the mean square error of the LMS algorithm. To simulate the behavior of the real system realistically, the measured vibration response of the machine's structure, $d(n)$, to a band-limited white noise excitation signal, $x(n)$, were used. The parameters were the filter length $L$ and the adaptation step size $\alpha$. The lower signal power threshold $P_{x, \min }$ was not investigated further because its sole purpose was to prevent algebraic instabilities. During the experiments, its value was set to $P_{x, \min }=10^{-38}$. Fig. 4 shows the results of the optimization process for the filter length $L$. It is evident that a higher filter length results in a more accurate model. However, because the number 